\title{
Le variazioni d'inclinazione e la sismicità che hanno preceduto il forte terremoto del Friuli del 6 Maggio 1976
}

\author{
8 \\ P. F. BIAGI $\left(^{*}\right)-$ P. CAIOI $\left(^{* *}\right)-$ MI. MIGANI (***) - M. C. SPADEA (****)
}

Ricevuto il 30 Sottembre 1976

Riassuxto. - In questa prima parte del lavoro, che ei proponiamo di dedicare alla disastrosa crisi sismica che ha colpito il Friuli nel 1976 , ci limitiamo a delineare le caratteristiche presismiche, che hanno preceduto la forte seossa del 6 Magrgio 1976.

Nelle premesse, vengono richiamati gli studi su fenomeni presismici, inziati in Italia già negli anni Cinquanta.

Sumarr. - In the first part of the work, which we have decided upon dedicating to the disastrous scismic erisis that struck the Friuli area in 1976 , wo will limit ourselves to delineating the pre-seismic characteristirs that preceded the strong shock of Nay 6th, 1976. In the introduction, reforence is to made to the studies of pre-seismic phenomena started in Italy in the 1950's.

\section{1 - Premesse}

1.1 - In un lungo lavoro sulle caratteristiche sismiche e geodinamiche della Val Parana - intesa in senso lato, come comprensiva della fossa arlrio-parlana e di tutti gli alvei e bacini dei fimmi che sfociano nellalto Adriatico, nonché dei monti che la limitano - fra

(*) Istituto di Geologia e Paleontologia, Enivorsità - Roma.

(**) Accademia dei Lincei - Roma.

(***) E.X.E.L., Compartimento Venezia - Venezia.

(****) Istituto Nazionale di Geofisiea - Osservatorio Geofisico Centrale Monte Porzio Catone - Roma. 
l'altro, a commento del diagramma delle energie sviluppate negli ultimi secoli fino a tutto il 1969, Caloi et al (1970)(7), con particolare riferimento all'ultimo secolo, osservano: "Esso inizia con il forte terremoto di Santa Croce, del 1875 che avera improvvisamente rotto la relativa calma sismica che durava in tutta l'ampia zona dal 1800. Alla disatrosa crisi sismica della zona del lago di Santa Croce, risponde, nello stesso ammo, ma potente attività sismica al lato opposto della pianura, dal mare, presso Cattolica, lungo le pendici appenniniche settentrionali. Sembra quasi che queste violente rotture dell'equilibrio elastico, ai due lati della pianura, siano legate come da causa ad effetto, e viceversa. Il fenomeno si ripeterà altre volte durante l'ultimo secolo; cosi, p. es., al forte terremoto adriatico del 1934 (Caloi, 1937) ('), farà riscontro, due anni dopo, il fort issimo teremoto del Cansiglio (Caloi, 1938) $\left({ }^{2}\right)$. Dal 1875, l'attivita sismica, in misura ridota, non ebbe praticamente più soste, manifestandosi, or qua or là, con scosse non molto intense, come se l'intero bacino padano fosse sotto l'azione perturbatrice di un'inquietudine tettonica unitaria, che si rivela presente soprattutto ai margini settentrionali e meridionali. Tale attività si ì andata fortemente attenuando nell'ultimo trentennio, accemnando ad una sensibile ripresa con il terremoto di VIII grado, verificatosi nel Delta il 30-XII1967. Non è eschuso che tale tregua trentenmale venga ulteriormente rotta con allre manifestazioni sensibili, probabilmente ai margini della zona ".

Previsione? No, naturalmente: soltanto un logico suggerimento della situazione, quale era venuta creandosi. Sta di fatto che, nell'anno successivo - il 1971 - forti scosse sismiche misero, a più riprese, in allarme le popolazioni rlell'Appennino morlenese ed emiliano in genere. Nel 1972, poi, ebbe inizio la drammatica crisi sismica che ha cosi duramente, e a lungo, colpito Ancona e zone limitrofe.

Ci si dovera aspettare la conferma dell'interdipendenza delle attività sismiche nelle due zone estreme. E ciò purtroppo, si è puntualmente verificato con la disastrosa erisi sismica del Friuli, iniziatasi la sera del 6 Maggio 1976.

1.2 - Va sottolineato il fatto che l'epicentro della forte scossa del Friuli (la $2^{\text {a }}$ ) del 6 Maggio 1976 , praticamente coincide con quello del terremoto dell'11. Oitobre 1954, verificatosi fra Bordano ed Osoppo. Com'e noto, quello del $195 \mathrm{t}$ costituisce il primo terremoto sorpreso nella fase di preparazione (Caloi, Sparlea, 1955) $\left({ }^{3}\right)$ : fu possibile, infatti, seguire la variazione della verticale apparente ad esso associata, dapprima lenta, quindi rapidissima, per tornare successivamente lenta e 
sfociare, dopo 22 giorni, nel terremoto. La scossa principale fu inoltre preceduta da tutta ma serie di microscosse, iniziatasi alcuni giorni prima di quella, a sua volta seguita da una lunga serie di repliche, alcume delle quali violente.

Altre avvisaglie presismiche, legate alla verticale apparente, si verificarono nella zona, associate a scosse manifestatesi nei dintorni di Tolmezzo fino al 1965; ma nessuna ebbe i caratteri inconfondibili della scossa del 1954 .

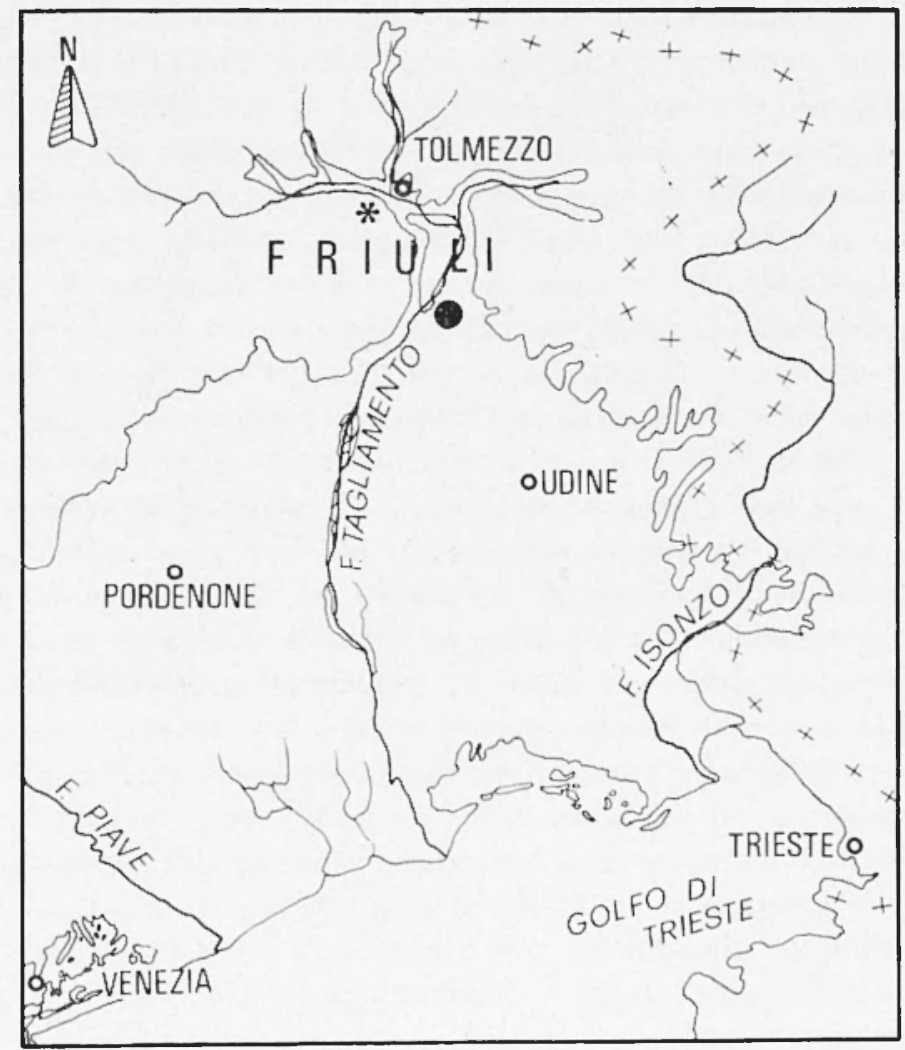

* Postazione clinografica

Epicentro

Fig. 1 - Epicentro del forte terremoto del 6 Magrio 1976 e postazione clinografica presso l'Ambinesta.

Figr. 1 - Epicenter of the Mas 6th, 1976 carthequalie and the tiltueter station installed at Ambiesta. 
1.3. - Ia non si limitarono alla verticale apparente e alla microsismicita le varazioni presismiche osservate in Italia. Fin dal 1950, Caloi, nelle indagini condotte per il rilevamento del modulo di elasticità nei pressi di grandi dighe e dei relativi bacini, aveva constatato che la microsismicità provocata determinava invariabilmente ma diminuzione del modulo di alasticità della roccia e, quindi, della velocità di propagazione delle onde sismiche, con particolare riferimento alle longitudinali. Di questi risultati, fu riferito in numerose pubblicazioni, in modo specifico, studiando il fenomeno della dispersione anomala nell'ambito delle altissime frequenze (Caloi, 1957) (i). Dopo un riesame delle cognizioni conseguite (Caloi, 1962) $\left(^{5}\right)$, di fronte all'agnosticismo, alla ostentata indifferenza, se non all'ostilitì, dell'ambiente direttamente interessato nei confronti delle nuove scoperte sul comportamento del campo elastico in roccia sollecitata da microsismicità naturale o provocata, e all'enorme interesse della loro estensione allo studio dei fenomeni sismici in generale, ai fini di ma possibile previsione, si c tornati sull'argomento con carattere vibratamente polemico, scrivendo fra l'altro (Caloi, 196t) $\left(^{\circ}\right)$ : "Si richiama, ancora una rolta, l'attenzione sull'interesse (a volte fondamentale, anche ai fini pratici) della costante osservazione delle variazioni del eampo slastico, nelle zone in cui un mezo solido è sottoposto " continue variazioni di temsione, comunque determinate. L'azione di tormento, associata alle rariazioni di tensione, si traduce in un decalimento det modulo elastico, testimoniato dal piü o meno semsibile diminuire della velocita di propagazione delle onde elastiche. L'osservazione continua del campo plastico in una zona sismica, combinata con la contemporanea ossereazione della varazione della vertienle apparente, può costituire nma valida via d'approceio mel problema della previsione dei teremoti".

Si e ritenuto opportuno fare questi richiami per dimostrare che, negli anni Cinquanta, in Italia si conducevano ricerche nel campo delle variazioni presismiche, con carattere di spiccata priorità (natulalmente all'insaputa degli ... italiani, quelli usi a guardare sempre oltre conline).

1.t. - Successivamente, inclagini del gencre furono iniziate anche allestero, particolarmente in Giappone, dove erano stati osservati mutamenti nella velocità delle onde sismiche, prima di grossi terremoti; mutamenti attribuiti a modilicazioni nelle proprieta elastiche della crosta terrestre, nell'area dove il terremoto stava preparandosi. ('iò avera indotto gli esperti grapponesi ar introdure, nel loro vasto pro- 


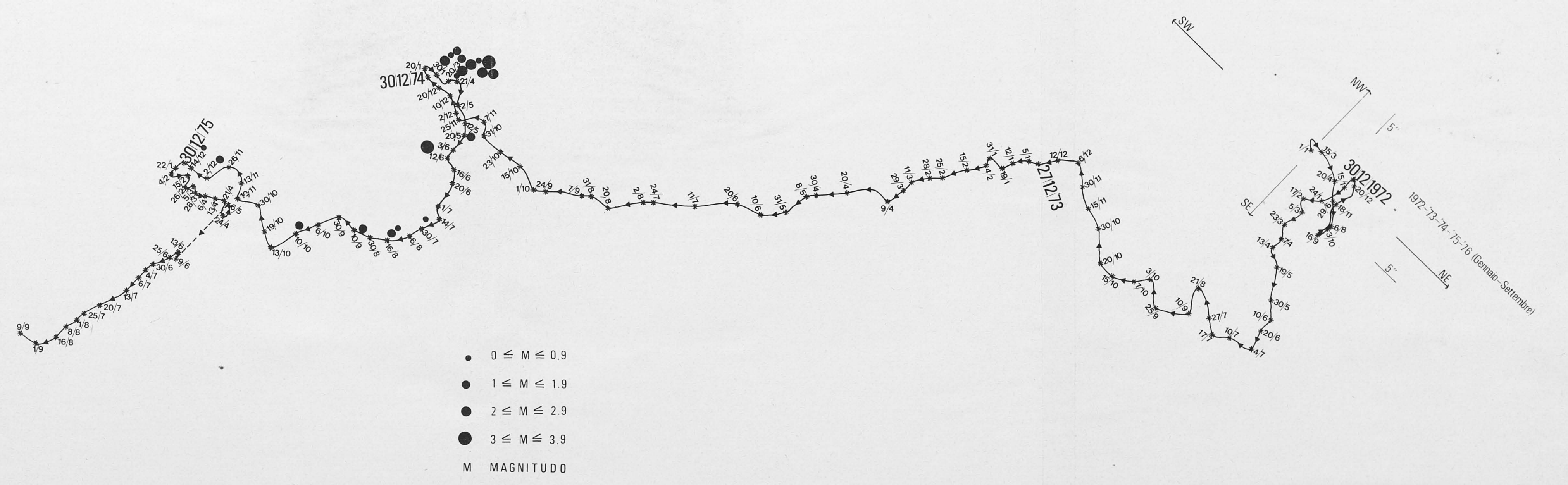

Fig. 2 - All'inizio del 1973 la verticale apparente inizia una decisa deviazione nei quadranti meridionali. Lenta durante tutto il 1973, essa tende ad assumere notevole velocità durante tutto il 1974 , con netta direzione
Sud. Verso la fine del 1974 la variazione della verticale tende a rallentare e a complicarsi in un cappio, in concomitanza con un periodo sismico, ch inizia bruscamente nella zona nel Marzo 1975, raggiunge il suo massim
nel Giugno 1975, per quindi diminuire d'intensità (fig. 3) gradualmente e lentamente, ed annullarsi nei primi mesi del 1976, contemporaneamente all'attenuarsi della vaniazione clinografica, cho praticamente si arresta necl'immediata vigilia del forte terremoto del 6 Maggio 1976. Andamento co-

Fig. 2 - At the beginning of 1973, the apparent vertical begin a decided deviation in the southern quadrants. Slow during all of 1953 trends to be quite rapid during all of 1974, in an overall southerly direc tion. Toward the end of 1974, the dip varation tends to dlow dow abruptly in the zone on March 1975 and reached its maximum in June 1975 , after which it gradually diminished in intensity (see Fig. 3) and ceased in the first months of 1976 . Contemporaneously, there was an attenuation of the tilting variation, which practicaliy disappeared at once before of

\begin{abstract}
the May, 6th 1976 strongest earthquake. Similar trend in
variation of the other parameters.
\end{abstract}


gramma di indagini, tese alla previsione dei terremoti (Tsuboi et al., $1962)\left({ }^{9}\right)$, anche periorliche determinazioni di velocità delle onde sismiche, provocate mediante esplosioni, in zone arl elevata sismicità opportumamente prescelte.

Ricerche analoghe si estesero arl altre nazioni. Nell'U.R.S.S. furono osservate variazioni del rapporto $\frac{v_{p}}{v_{s}}$ nella zona di Garm, prima rli alcuni terremoti, con magnitudo variabile da 4.2 a 5,4. Negli U.S.A. si inrlagò particolarmente sulle arrisaglie presismiche che averano precerluto il ristruttivo terremoto di San Fernando del 9 Febbraio 1971, riscontrando una ben delineata anomalia nel rapporto $\frac{v_{p}}{v_{s}}$. Altre ne seguirono in Giappone e negli U.S.A. Il fenomeno legato alla variazione del rapporto $\frac{v_{p}}{v_{s}}$, negli Stati Uniti fu spiegato con l'espansione (dilatancr) che si verificherebbe nella fase di preparazione di un terremoto, sebbene questa venisse attribuita arl eventi fisicamente discutibili.

Le ricerche furono quindi estese alle variazioni presismiche associate ai movimenti crostali, alla resistività elettrica, al campo magnetico, all'emissione rarlon, ecc. Tali ricerche sono state recentemente riassunte da uno di noi (Caloi, 1976) $\left(^{8}\right)$.

Sulla base dei risultati finora raggiunti nell'U.R.S.S. e negli U.S.A., era stato tracciato un rliagramma che dà lintervallo di tempo precursore (espresso in giorni, in scala logaritmica) in funzione rlella magnitudo. Ebbene, con esso si concorla ottimamente l"intervallo precursore del terremoto del Friuli del 1954 di magniturlo 4.4, primo studiato da questo punto di vista (fig. 4).

2 - VARIAZONI PRESISMICIIE RISCONTRITE IN CORRISPONDENZA JUEL FORTE TERREMOTO DEI FrIVLI DEL 6 MIAGio 1976.

2.1. - Liepicentro della scossa disatrosa del 6 Maggio 1976 fu reterminato (sia pure in via provisoria) usufruendo delle cinque stazioni dell" ENEI, più vicine: Ambiesta (Tolmezzo), I a Maina (Sauris), Pieve di Carlore, Vajont e Mis (Belluno).

Le determinazioni fornite dai Centri internazionali sono sempre alquanto approssimative, con particolare riguardo alla profondita, specie per terremoti della crosta terrestre. I motivi sono evidenti e si irlentificano principalmente con le inomogeneità diffuse e le varia- 
zioni degli spessori della crosta nelle diverse direzioni. Ciò sa bene chi si occupa di questi problemi, ed ha imparato, a sue spese, a diffidare dei risultati ottenuti, arralendosi di stazioni troppo discoste clagli epicentri, e a limitare le inclagini sulle coordinate ipocentrali servendlosi dei tempi delle stazioni prossime.

Le coordinate epicentrali risultarono come segue:

$$
\varphi=46^{\circ} 16^{\prime}, 5 \mathrm{~N} ; \quad \lambda=13^{\circ} 06^{\prime}, 0 \mathrm{E}
$$

con riferimento alla $2^{a}$ scossa. La profondità è risultata non inferiore ai $15 \mathrm{~km}$ e il tempo origine

$$
H=20.00 .15,5
$$

Altri hanno ottenuto per queste grandezze i valori più disparati, specialmente per quanto si riferisce al tempo origine, che viene persino dato 7 secondi prima di quello riportato. Se così fosse, poiché il tempo della $2^{*}$ scossa all' $A$ mbiesta - a $15 \mathrm{~km}$ di distanza epicentrale - $\mathrm{fu}$ per le $P_{g}$ di $20.00 .18,5( \pm 2 / 10$ di sec), le onde longitudinali dirette avrebbero impiegato ben $10 \mathrm{sec}$ a compiere il tragitto di $20 \mathrm{~km}$ ca.: il che ì manifestamente assurdo. Per la magnitudo, si è scelto un ralore compreso fra 6.3 e 6.5 .

Esula, per ora, dalla nostra attenzione il problema del meccanismo del terremoto all'ipocentro, da altri tratto sulla base delle repliche, il che non i aflatto corretto. Ci riserviamo di ritornare sull'argomento, quando potremo usufruire delle registrazioni originali di un congruo numero di stazioni sismiche, sparse per il monclo.

Qui ei limitiamo a riferire sulle variazioni presismiche, osservate nella zona dell'Ambiesta.

2.2. - Si tratta, naturalmente, di un'inclagine a posteriori: le stazioni clinografiche funzionanti presso le dighe "ENEI, hanno infatti uno scopo di controllo del tutto locale, con particolare riferimento alla stabilità degli sbarramenti e alla stabilità della culla rocciosa, sopportante a monte il bacino iclrico.

Non tutte le postazioni clinografiche sono atte al rilievo di fenomeni di carattere tettonico. Si prestano invece a questo scopo, le postazioni a lato della diga dell'Ambiesta, con particolare riferimento a quella posta al largo della sponda sinistra, sul bordo di una faglia attiva.

Questa postazione ì particolarmente sensibile alle manifestazioni sismiche locali; sicche molto raramente le variazioni della verticale 
ivi osservate sono trascurabili. Per esempio, durante il 1971, l'attività clinografica fu piuttosto vivace, associata com'era ad una serie di scosse sismiche, di piccola intensità, verificatasi nella zona.

Il 1972, invece, fu un anmo relativamente tranquillo, esente da attività sismica locale: i lievi e disordinati movimenti osservati, essendo da attribuire all'azione perturbante di depressioni bariche in transito.

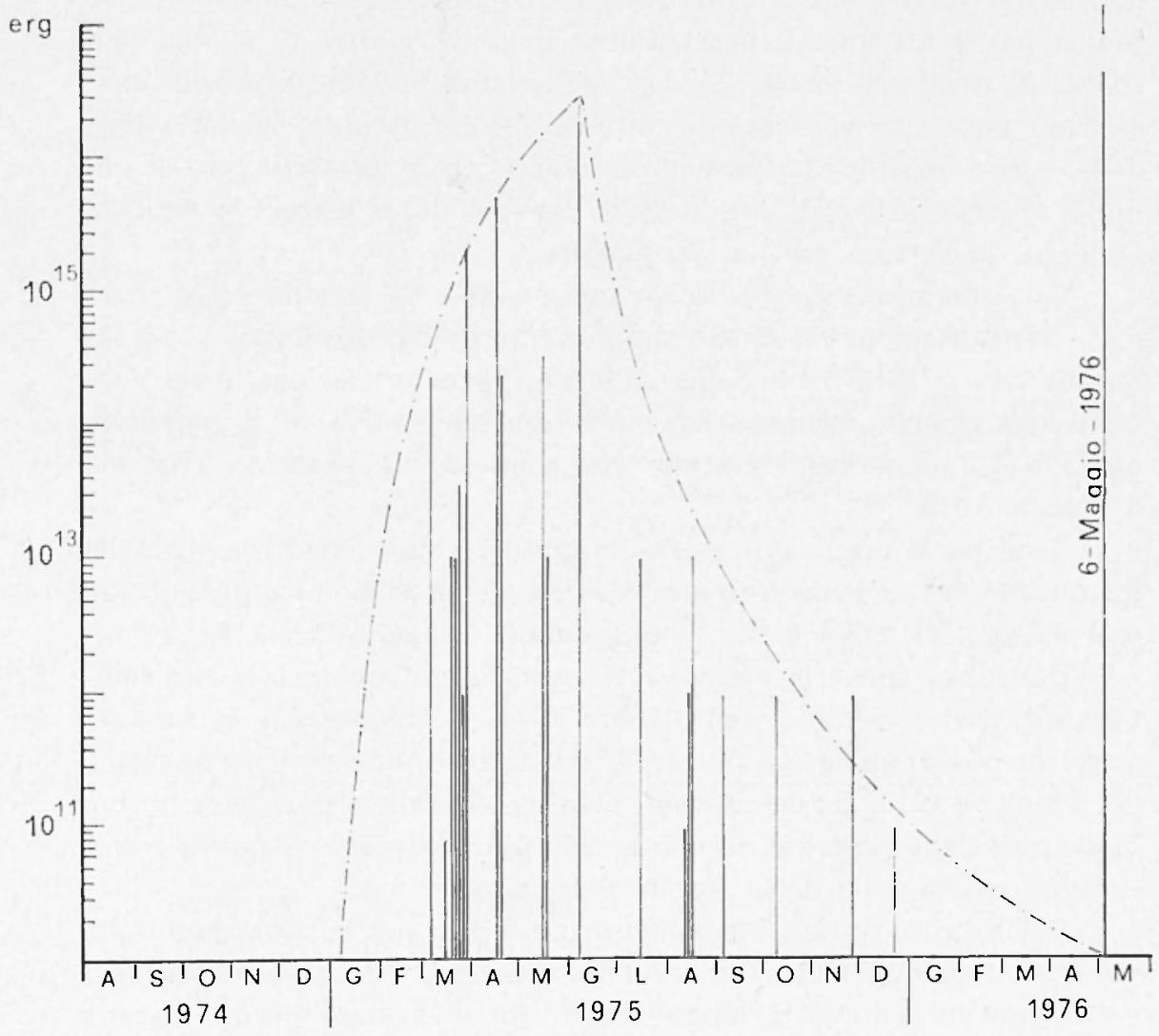

Fig. 3 - Andamento dellattivita presismica che ha preceduto il forte terremoto del Frinli del 6 Magrgio 1976 (v. figr. 2).

Fig. 3 -- Trend of the prescismic activity that preceded the strong frinli earthqualie of Maly (th, 1976 (c.f. Fig. 2). 
Con il 1973, il fatto nuovo (fig. 2). Con qualche esitazione, la rerticale apparente piega, decisa, nel settore di $\mathrm{SE}$, e compie, sia pure lentamente, un'ampia, contrastata voluta fino al 6 Dicembre. A partire da questo giorno, la variazione della verticale volge lecisamente verso SSE e, in detta direzione, procerle veloce per quasi tutto il 1974. Ia sensibile deviazione osservata negli ultimi due mesi, complicatasi ardirittura con un cappio, lasciava prevedere un movimento tettonico in atto. Questo, infatti, cominciò a manifestarsi con una prima scossa il 7 Marzo 1975 e, con alterna intensità, si fece particolarmente intenso fra il 2+4 Marzo e l's Giugno 1975 , giomo in cui la zona fu messa in allarme da ma scossa di magnitudo 3.6 ca. Indi l'inquietudine tettonica parve attenuarsi, per esaurirsi il 25 Dicembre 1975 , con una scossa di carattere locale. Ia fig. 3 riassume l'andamento delle magnitudo delle scosse di questo periodo. Nel frattempo, la variazione della verticale andava riducendosi sempre più e praticamente si annullava verso la line di Aprile 1976. Ta sera del 6 Maggio si verilico, violenta, la rottur'a del campo elastico.

Va sottolineata la stretta analogia con la variazione della verticale, verificatasi in occasione del terremoto del Friuli del 1954: in questo caso, lenta variazione all'inizio, lenta variazione nella fase finale, variazione veloce nella parte centrale; salve le proporzioni, analogo il comportamento che ha condotto alla cruenta crisi del 6 Magrgio 1976.

Come per la precedente scossa, la durata è stata condizionata dalla magnitudo del veniente terremoto: 22 giomi nel primo caso (magnitudo dellordine 4.4), circa 3 anni nel secondo (magnitudo fra 6.3 e 6.5 ).

Del resto, anche le prescosse si verificarono con successione comparabile. Nel terremoto dell'Ottobre 1954 le microscosse si manifestano in prossimita della 2 a fase di rallentamento; per la forte scossa del 6 Maggio 1976, la fase presismica anticipa il rallentamento definitivo della rariazione clinografica, con la sola differenza che - questa volta - sono scosse autentiche, sentite dalla popolazione.

Del resto, ì questo l'andamento osservato per le variazioni della velocita, massime nella fase centrale dellanomalia presismica.

A questo punto, ci siamo chiesti se - avendo potuto seguire passo passo landamento delle perturbazioni presismiche - saremmo stati in grado di predire, con qualche margine di sicurezza, ciò che stara maturauclo. In coscienza, la risposta è negativa. Troppi sono ancora gli elementi d'incertezza che condizionano il maturare di siffatti fenomeni; e di essi si fa cenno nel recente lavoro di mo di noi (Caloi, 1976) $\left.{ }^{8}\right)$. 


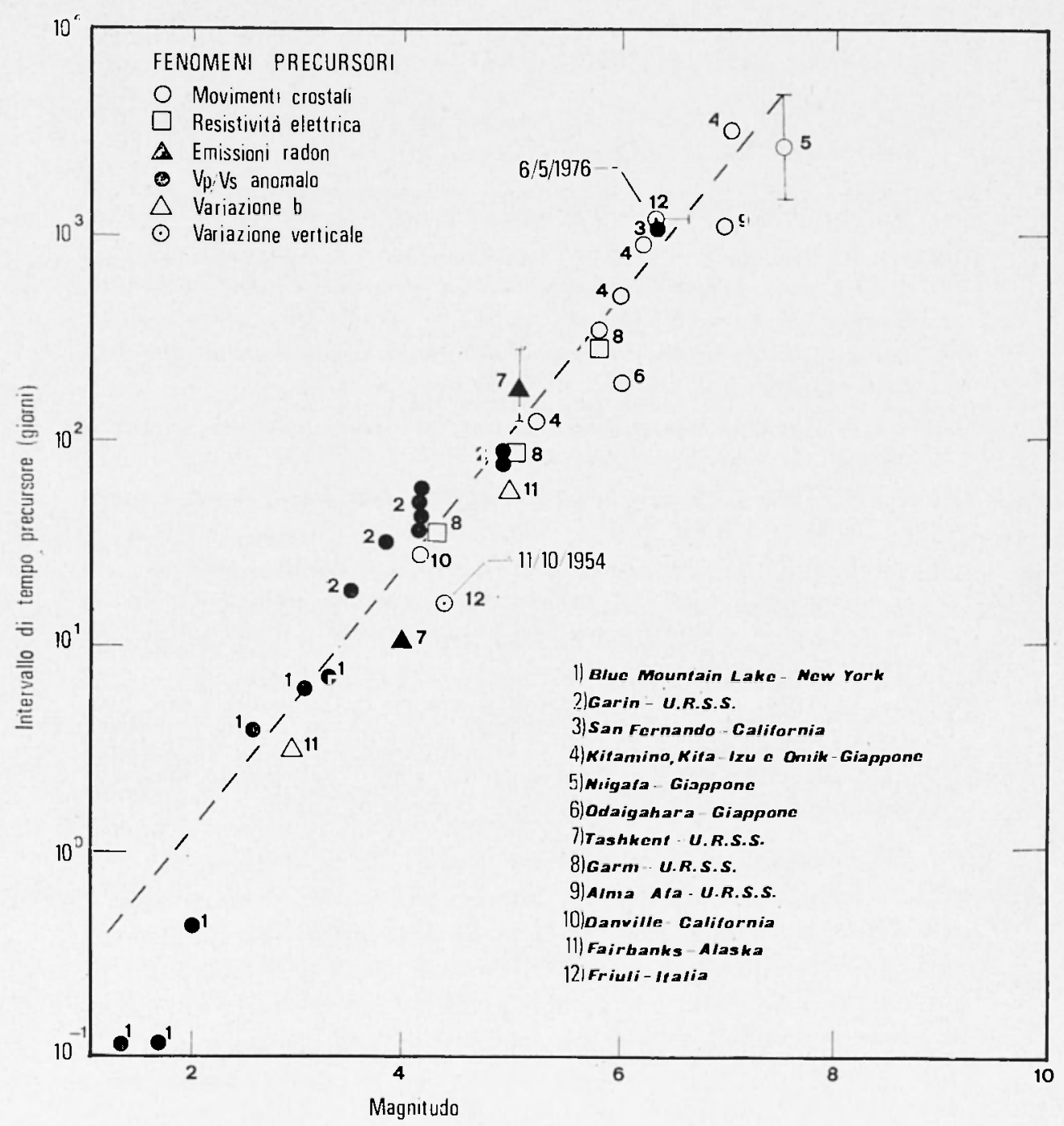

Fig. 4 -- Il terremoto del Friuli del 1954 fu il primo seguito nelle sue variazioni presismiche, clinografica e microsismografica. La durata della variazione presismica e la sua magnitudo concordano bene con il diagramma tracedato circa vent anni dopo. Con questo diagramma concorda pure la variazione presismica del forte terremoto del Friuli del 1976, ritenuto di magnitudo 6.3. Nel caso di $y=6.5$ (come è stata calcolata da qualehe centro internazionale), il cerehietto verrebbe a cadere proprio sulla retta mediana.

Fig. 4 - The Friuli earthquake, 1954, was the first studied in its pre-seismic variations, tilting and microseismic. The duration of the pre-seismie raviation and its magnitude agrees well with the diagram drawn about 20 years later. The pre-seismic variation in the Friuli earthquake of May 6 th, 1976 considered of magnitude 6.3 al., is in well aceordance with this diagram. In the case of $M=6.5$ (as has heen caleulated by some internatio. nal eenters), the circle would fall precisely on the middle straight line. 


\section{BIBIAOGRAFIA}

(1) Catol P., 1937. - Il teremolo adrialico del 30 Novembre 1931. "Boll. Soc. Sismol. Ital. ", XXXV.

) Canor P., 1938. - Ricerche su lerremoli ad origine vicina. Scosse del Cansiglio dellOllobre 1936. "Lal Riccrea soientifica ", S. II, A. IX, II.

(3) CA.oI P', SPABEA M. C., 1955. - Relazioni fra lenle variazioni dinclimazione e moli sismici in zone ad elevala sismicila. "R(nd. Ace. Naz. Linceci "s. SIII, XVIII.

(1) Cano1 P., 1957. - Sulla dispersione delle onde sismiche nell ambilo delle allissime frequenze. "Amuali di Goofisica ", X, 3-4.

$\left(^{5}\right)$ Caroi I’, 1962. - Aspelli della dinamica di rocce, calcestruzzo ed acque. "Annali di Geofisica", XV, 2-3.

$\left.{ }^{6}\right)$ Canor P., 1964. - La geodinamiea al servizio delle grandi dighe. "Annali di Geofisical", XVI, 1.

(7) CAmor P., Romund) G., SPADEA M. C., 1970. - Caralleristiche sismiche e geodinamiche della Tal Padana quali risullano dall altivilà sismica ivi verificalasi dallinizio dell Era Tolgare a lullo il 1969. "Annali di Geofisical ". XXIII, 2-3.

$\left(^{8}\right)$ CAlor P'., 1976. - Sulle possibilità di previsione dei lerremoli. "Ace. Naz. dei Lincei: Commiss. di studio delle calamità naturali ..." XIV.

(9) Tsubor Ch., Wabati K., Hagiwara 'T., 1962. - Prediclion of Earllhquakes. "Liuth. Res. Institute", Tniversity of Tokyo. 